\title{
Efectos de la Radiación Ultravioleta B (UVB) SOBRe DIFERENTES VARIEDADES DE QUINOA. I. EFECTOS SOBRE LA MORFOLOGÍA EN CONDICIONES CONTROLADAS
}

\author{
M. L. PEREZ1 ${ }^{1}$ J. A. GONZÁLEZ² y F. E. PRADO1
}

\begin{abstract}
Resumen: Se describen los efectos de la radiación UV-B (RUV-B) sobre algunos parámetros de crecimiento: altura de la planta (A), diámetro de tallo (DT), largo $x$ ancho (LA), número de hojas (NH), área foliar específica (AFE) y masa foliar específica (MFE) en cinco variedades de quinoa. Los efectos de la UV-B fueron diferentes según la variedad y parámetro considerado. Así, A se incrementó en las variedades CICA $(P \leq 0,04)$ y Robura $(P \leq 0,02)$; mientras DT fue influenciado positivamente en CICA $(P \leq 0,0002)$ y Faro Roja $(P \leq 0,017)$. LA sólo mostró cambios significativos $(P \leq 0,05)$ en CICA. El NH fue la variable que experimentó cambios positivos en todas las variedades, observándose los más pronunciados en Faro Roja $(P \leq 0,003)$, CICA $(P \leq 0,003)$ y Ratuqui $(P \leq 0,015)$. La MFE cambió positivamente en Faro Roja, Kancolla y Robura $(P \leq 0,05)$. CICA fue la única variedad que experimentó incrementos significativos en todos los parámetros evaluados, seguida de Faro Roja y Robura. El menor porcentaje de cambios ocurrieron en Kancolla y Ratuqui. Las variaciones observadas se discutieron en términos de adaptación evolutiva.
\end{abstract}

Palabras clave: Radiación UV-B, Chenopodium, crecimiento.

Summary: Effects of ultraviolet UV-B (UVB) on different varieties of quinoa. I. Effects on morphology under controlled conditions. The effects of UV-B radiation (RUV-B) on growth parameters: plant height $(H)$, stem diameter $(S D)$, length $x$ wide $(L W)$, leaf number $(L N)$, specific leaf area $(S L A)$ and specific leaf mass (SLM) of five quinoa varieties are described. RUV-B effects were different according to analyzed variety and parameter. The $\mathrm{H}$ was increased in $\operatorname{CICA}(P \leq 0,04)$ and Robura $(P \leq 0,02)$ varieties, while SD was increased in CICA $(P \leq 0,0002)$ and Faro Roja $(P \leq 0,017)$ varieties. The LW changed significantly in CICA $(P \leq 0,05)$ variety only. The LN showed positive changes in all quinoa varieties exposed to RUV-B. Highest changes were found in Faro Roja $(P \leq 0,003)$, CICA $(P \leq 0,003)$ and Ratuqui $(P \leq 0,015)$ varieties. The SLM positively changed in Faro Roja, Kancolla and Robura varieties $(P \leq 0,05)$. The CICA variety showed significant increases in all evaluated parameters, followed by Faro Roja and Robura varieties. Less parameter changes occurred in Kancolla and Ratuqui varieties. The observed changes were discussed in terms of adaptive evolution.

Key words: UV-B radiation, Chenopodium, growth.

\section{INTRODUCCIÓN}

Quinoa es una especie originaria de los andes sudamericanos y posee una distribución altitudinal y latitudinal amplia (Risi \& Galwey, 1984). En efecto, su desarrollo abarca una extensa área a lo

${ }^{1}$ Facultad de Cs. Naturales e IML, Universidad Nacional de Tucumán, Cátedra de Fisiología Vegetal, Miguel Lillo 205, 4000 Tucumán.

${ }^{2}$ Fundación Miguel Lillo, Instituto de Ecología, Miguel Lillo 251, 4000 Tucumán. largo de la Cordillera de los Andes entre Ecuador y Sur de Chile La plasticidad genética de esta especie le ha permitido adaptarse y crecer desde alturas superiores a $3600 \mathrm{~m}$ s.n.m hasta casi el nivel del mar como ocurre en Brasil, Perú, Francia, España, Inglaterra, Italia, Dinamarca, Estados Unidos y Egipto, entre otros. Incluso es capaz de prosperar en terrenos marginales con escasa potencialidad agrícola, que no resultan aptos para los cultivos tradicionales (Burnouf-Radosevich, 1988; González \& Prado, 1992; Ruffino et al., 2010). Este hecho ha motivado que su cultivo se halla expandido notablemente en los últimos 
tiempos, siendo ensayada y estudiada en distintas regiones del mundo. A la versatilidad de su cultivo debe añadirse su alto valor alimenticio, derivado de sus propiedades nutritivas que resultan únicas entre los alimentos de origen vegetal (González et al., 1989, 2010, 2012; Prado et al., 2010).

La plasticidad de la quinoa para desarrollarse en una gran amplitud de ambientes, implica adaptaciones tanto fisiológicas como morfológicas que varían de una variedad a otra. Una de las adaptaciones más notables que exhibe la quinoa es su extraordinaria tolerancia a la radiación solar, específicamente a la ultravioleta B (UVB), como lo han demostrado diferentes estudios llevados a cabo en condiciones naturales y en ambientes controlados (Palenque et al., 1997; Sircelj et al., 2002; Hilal et al., 2004; González et al., 2009; Prado et al., 2011). Sin embargo, la mayoría de estas investigaciones se centraron a nivel celular, fundamentalmente en relación a parámetros bioquímicos (producción de metabolitos primarios y secundarios), sin profundizar en los aspectos macroscópicos referidos a planta entera. Por lo tanto, el objetivo de este trabajo fue estudiar el efecto de la radiación UV-B (RUV-B) sobre determinados parámetros morfológicos (altura de la planta, diámetro de tallo, área foliar, número de hojas y masa foliar específica) en variedades de quinoa de distintos orígenes altitudinales, a fin de precisar el grado de afectación de los mismos y de este modo contribuir a la selección de variedades mejor adaptadas.

\section{Materiales y Métodos}

Se utilizaron semillas de cinco variedades de quinoa, cuatro de ellas originarias del altiplano Peruano-Boliviano (3.500-3.700 m s.n.m.): CICA y Kancolla (Perú), Ratuqui y Robura (Bolivia) y una originaria de Chile con hábitat de crecimiento a nivel del mar: Faro Roja. Las semillas se sembraron en macetas plásticas de $10 \mathrm{~cm}$ de diámetro conteniendo suelo orgánico. Para cada variedad se sembraron 10 macetas. Una vez completada la emergencia de las plántulas se realizó un raleo manual hasta obtener 5 plántulas por maceta, equidistantemente distribuidas. Luego del raleo las macetas fueron trasladadas a una habitacióninvernadero a $25^{\circ} \mathrm{C}, 60 \%$ de humedad relativa, 500 $\mu \mathrm{mol} \mathrm{m} \mathrm{m}^{-2} \mathrm{~s}^{-1}$, suministrados por tubos fluorescentes de $40 \mathrm{~W}$ (Philips, Argentina), y fotoperiodo de 12 h. Las macetas se regaron diariamente con agua destilada a fin de minimizar los riesgos de estrés hídrico. A los 53 días de cultivo (altura de la planta $15-20 \mathrm{~cm}$ ) las macetas se dividieron en dos grupos denominados control y UV-B, respectivamente. El grupo fue irradiado durante 17 días con RUV-B, suministrada por lámparas fluorescentes que emiten en la zona UV-B del espectro lumínico (pico máximo de emisión: $312 \mathrm{~nm}$ ) (Q-Panel, Cleveland, USA). La RUV-B se aplicó durante 10 min por día y corresponde a una irradiancia diaria de $7,5 \mathrm{~W} \mathrm{~m}^{-2}$ (a nivel de follaje); equivalente a la RUV-B solar que incide sobre las plantas en un día soleado de verano en San Miguel de Tucumán. La RUV-B se midió con un radiómetro portátil provisto de sensor para UV-B (Cole Parmer, USA). Las aplicaciones de RUV-B se realizaron al mediodía para evitar cualquier variación de tipo circadiano en los parámetros a medir. Durante todo el ensayo las fuentes de iluminación (visible y UV-B) se mantuvieron a $60 \mathrm{~cm}$ por encima del follaje de la plántulas. Para evitar cualquier filtración de RUV-B el grupo de macetas UV-B se mantuvo separado del grupo control por un plástico de color negro de 100 $\mu \mathrm{m}$ de espesor, opaco a la RUV-B.

Se calcularon los siguientes parámetros morfológicos:

Altura de la planta: Se registró diariamente a partir de la apertura cotiledonar ( $5^{\text {to }}$ día después de la siembra) y se continuó hasta la finalización del ensayo. Para las mediciones se usó una cinta métrica graduada al mm.

Diámetro de tallo: Se procedió como en el caso anterior, pero usando un calibre digital.

Largo por ancho: Se determinó para el primer par de hojas verdaderas. Para la medición del largo y ancho de la hoja se utilizó un calibre digital.

Número de hojas: Se realizó por conteo manual.

Para cada variedad se utilizaron 10 plantas diferentes a fin de obtener una mejor aproximación al valor real del parámetro medido.

Área Foliar Específica: El AFE se determinó al finalizar las aplicaciones de RUV-B. Para ello se seleccionaron hojas maduras, ubicadas en la parte superior del follaje. Mediante un sacabocados se extrajeron discos de $3 \mathrm{~mm}$ de diámetro de la parte central de la lámina (aproximadamente 8 discos por hoja). Los discos se secaron en estufa a $84{ }^{\circ} \mathrm{C}$ por 24 
$\mathrm{h}$ a fin de obtener el peso seco (PS). Con los valores de PS se procedió a determinar el AFE y 1/AFE que corresponde a la masa foliar específica (MFE). Para la determinación del AFE se utilizaron 5 plantas distintas para cada variedad, en ambos grupos: control y UV-B.

Para los análisis estadísticos se aplicó la prueba paramétrica $t$ de Student para un nivel de probabilidad de $(P<0,05)$.

\section{Resultados}

Altura de la planta (A): En la Fig. 1 se muestra la variación en la altura de la planta para todas las variedades ensayadas. La RUV-B ejerció un efecto estimulante significativo sobre A en las variedades CICA $(P \leq 0,04)$ y Robura $(P \leq 0,02)$; el resto de las variedades, por su parte, no fue afectado por la RUV-B.

En coincidencia con nuestros resultados, en experiencias llevadas a cabo en la ciudad de La Paz (Bolivia), se demostró la inducción de un efecto estimulante de A por la RUV-B solar en las variedades Chucapaka, Robura y Sayaña (Palenque et al., 1997).

Diámetro de tallo (DT): En la Fig. 2 se muestra la variación del diámetro de tallo en todas las variedades ensayadas.

Tanto en la variedad CICA como en Faro Roja el DT aumenta significativamente $(P \leq 0,0002$, CICA) y $(P \leq 0,017$, Faro Roja) por efecto de la RUV-B cuando se compara con las plantas control, siendo más marcado en la segunda. Los incrementos observados al final del período experimental fueron $62 \%$ y $18 \%$ para Faro Roja y CICA respectivamente. Las restantes variedades no fueron afectadas significativamente por la RUV-B.

Largo por ancho (LA): En la Fig. 3 se muestra la evolución de LA correspondiente al primer par de hojas verdaderas durante el tratamiento con RUVB.

Estadísticamente la variedad CICA fue la única que resultó afectada en el parámetro LA por la RUVB mostrando un leve incremento en relación a las plantas no expuestas a la RUV-B $(P \leq 0,05)$. En las restantes variedades no se observaron efectos significativos de la RUV-B sobre LA.
Número de hojas (NH): La Fig. 4 muestra el efecto de la RUV-B sobre el número total de hojas al final del período experimental.

Se observa que en todas las variedades la RUV-B indujo un incremento en el número de hojas. Los mayores incrementos de $\mathrm{NH}$ se observaron en las variedades Faro Roja, CICA y Ratuqui [33 $\%(P \leq 0,003), 32 \%(P \leq 0,003)$ y $14,3 \%(P \leq$ $0,015)$, respectivamente]; mientras que los más bajos correspondieron a las variedades Robura y Kancolla. Resulta interesante destacar que el efecto inductor mostró un desfase temporal en estas variedades ya que el mismo se hizo evidente a partir del día 14 en Faro Roja, día 21 en CICA, día 28 en Ratuqui y día 58 en Robura y Kancolla.

Área foliar específica (AFE) y masa foliar específica (MFE): La Tabla 1 muestra las variaciones en los valores de AFE y MFE de distintas variedades de quinoa inducidos por la exposición de las plantas a la RUV-B artificial. En Faro Roja y Kancolla la RUV-B tuvo un efecto positivo sobre el AFE, mostrando ambas variedades un incremento significativo en el valor de dicho parámetro; en tanto que, la variedad Robura mostró un comportamiento opuesto ya que el valor del AFE resultó disminuido por la RUV-B. Las variedades CICA y Ratuqui también presentaron disminuciones del AFE, pero no fueron estadísticamente significativas. El valor de MFE, que corresponde a la inversa del AFE, mostró un comportamiento similar pero en sentido opuesto es decir, resultó significativamente disminuido en las dos primeras variedades y significativamente aumentado en la última. Al igual que en el AFE las variaciones de MFE en las variedades CICA y Ratuqui no fueron estadísticamente significativas (Tabla 1).

\section{Discusión}

Comparando las respuestas morfológicas de las distintas variedades de quinoa a la RUV-B se pudo observar que, a excepción de $\mathrm{NH}$, los parámetros analizados resultaron afectados solo en algunas variedades. Por otra parte, las respuestas no fueron uniformes en cuanto a intensidad y variedad en la que se manifiesta (Figs. 1-4). En relación a NH, el único de los parámetros testeados que fue afectado 
Bol. Soc. Argent. Bot. 50 (3) 2015
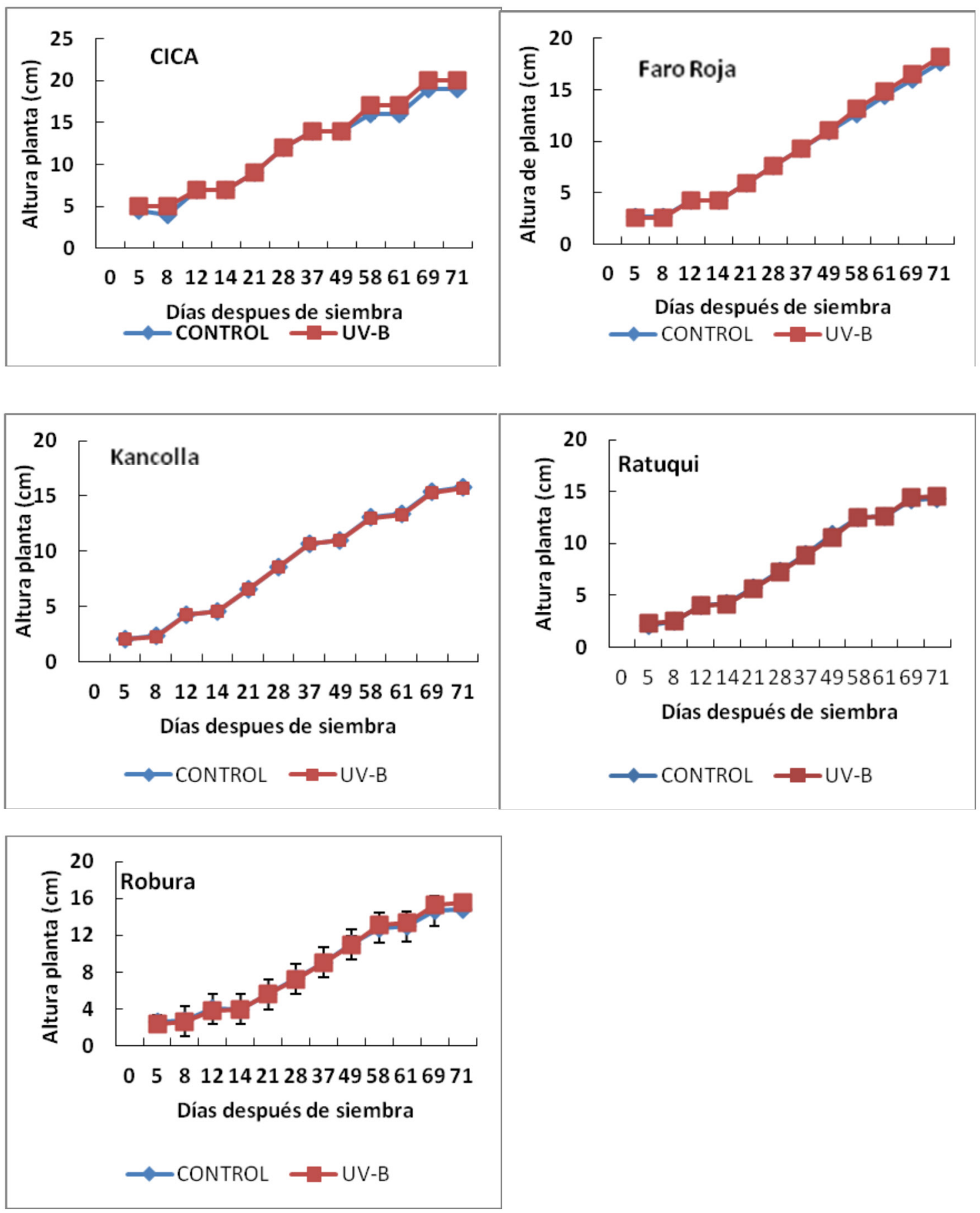

Fig.1. Efecto de la radiación ultravioleta B (UV-B) sobre la altura de la planta. Cada punto representa la media de 10 plantas. La barra en cada punto indica la DS. Donde no se nota la barra es porqué la misma cae dentro del valor promedio. 

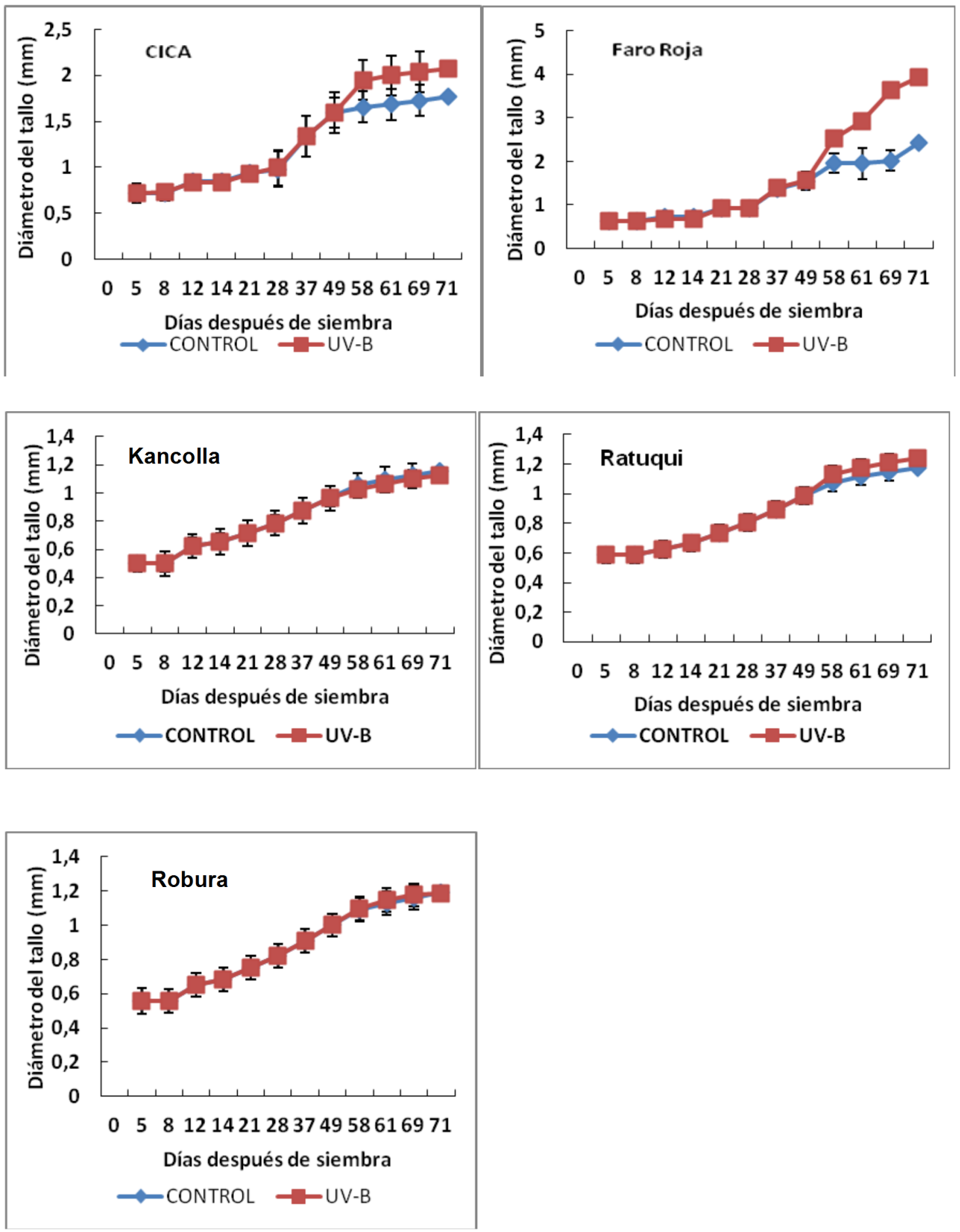

Fig. 2. Efecto de la radiación ultravioleta $B$ (UVB) sobre el diámetro del tallo principal. Cada punto representa la media de 10 plantas distintas. La barra en cada punto indica la DS. Donde no se nota la barra es porqué la misma cae dentro del valor promedio. 
Bol. Soc. Argent. Bot. 50 (3) 2015
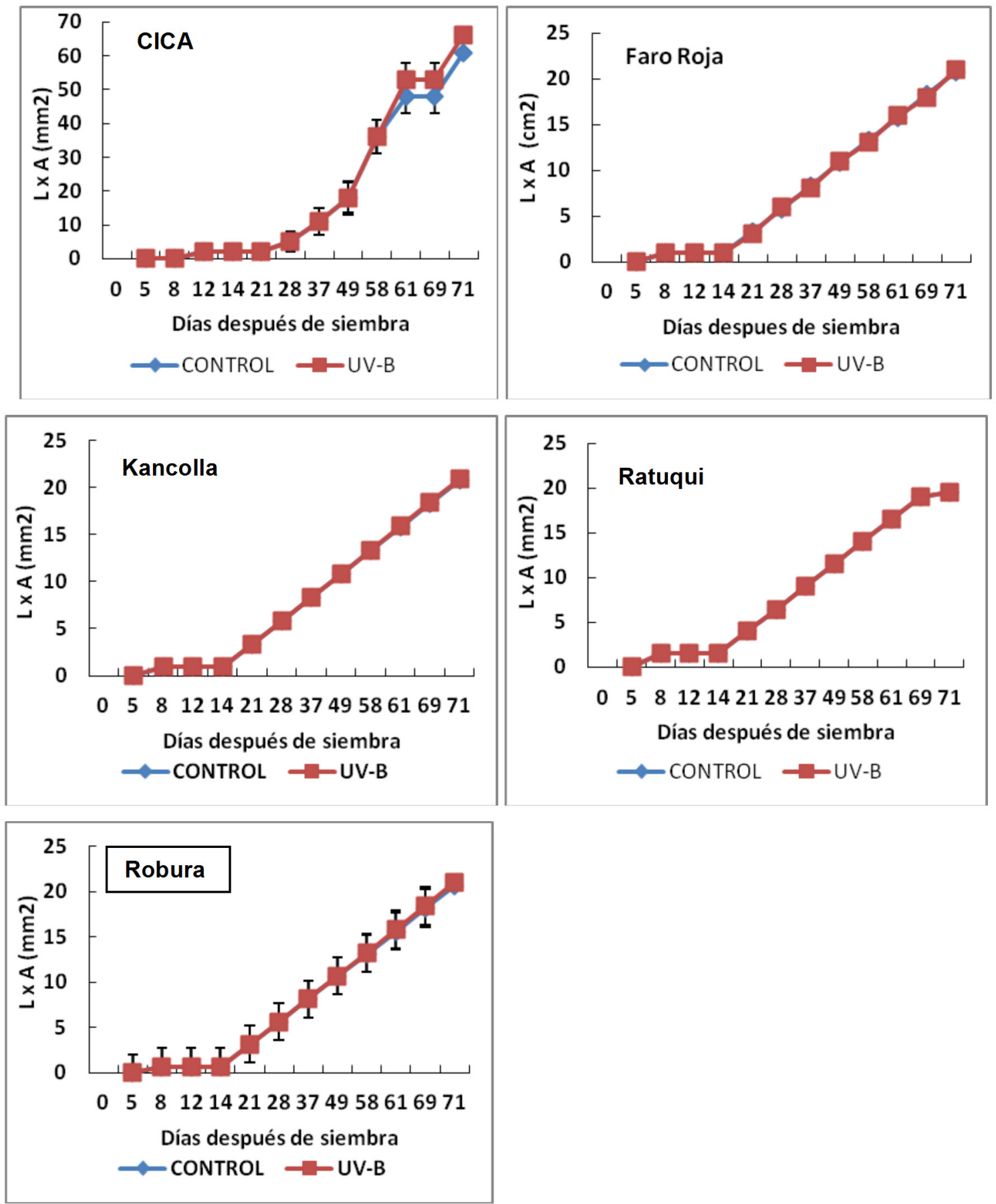

Fig. 3. Efecto de la radiación ultravioleta $B$ (UVB) sobre el producto largo (L) $x$ ancho (A) del primer par de hoja verdadera. Cada punto representa la media de 10 plantas distintas. La barra en cada punto indica la DS. Donde no se nota la barra es porqué la misma cae dentro del valor promedio. 

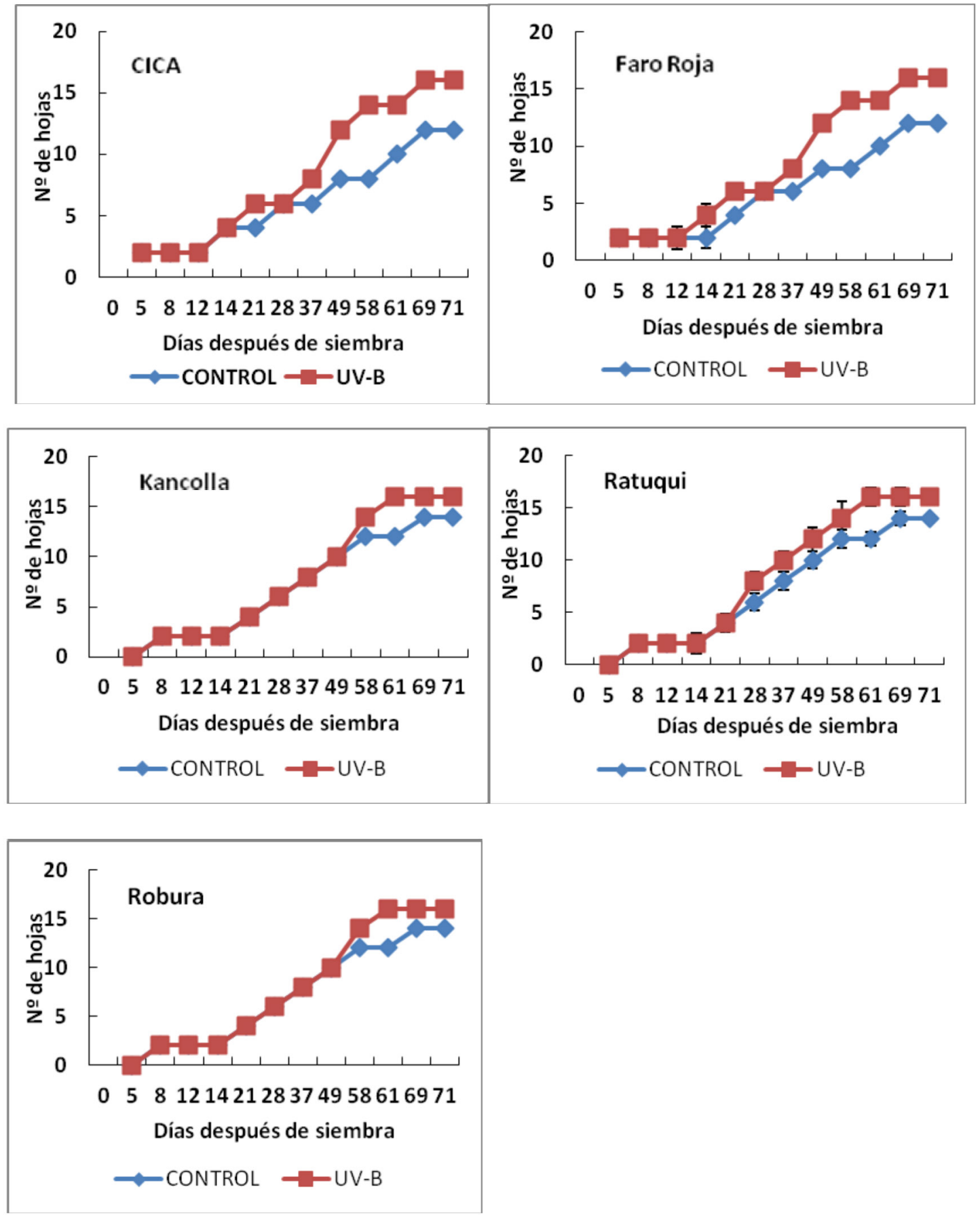

Fig. 4. Efecto de la radiación ultravioleta $B$ (UVB) sobre el número de hojas verdaderas desarrolladas hasta el final de la experiencia. Cada punto representa la media de las hojas presentes en 10 plantas distintas. La barra en cada punto indica la DS. Donde no se nota la barra es porqué la misma cae dentro del valor promedio. 
Bol. Soc. Argent. Bot. 50 (3) 2015

Tabla 1. Efecto de la radiación ultravioleta B (UVB) sobre la MFE.

\begin{tabular}{|lcccc|}
\hline Cica & $\begin{array}{c}\text { AFE }(\text { Control) } \\
(\mathrm{cm} 2 / \mathrm{gPS})\end{array}$ & $\begin{array}{c}\text { AFE (UVB) } \\
(\mathrm{cm} 2 / \mathrm{gPS})\end{array}$ & $\begin{array}{c}\text { 1/AFE - control } \\
(\mathrm{mgPS} / \mathrm{cm} 2)\end{array}$ & $\begin{array}{c}\text { 1/AFE - UVB } \\
(\mathrm{mgPS} / \mathrm{cm} 2)\end{array}$ \\
Faro Roja & $438,9(44,4)$ & $346,1(61,9)$ & $2,278(0,25)$ & $2,889(0,4)$ \\
Kancolla & $340,1(45,6)^{*}$ & $445,9(42,8)^{\star}$ & $2,940(0,42)^{\star}$ & $2,242(0,20)^{\star}$ \\
Ratuqui & $368,4(49,4)^{\star}$ & $393,8(59)^{*}$ & $2,714(0,43)^{\star}$ & $2,539(0,39)^{*}$ \\
Robura & $497,2(45,3)$ & $421,6(82,2)$ & $2,011(0,18)$ & $2,371(0,52)$ \\
\hline
\end{tabular}

$\left(^{*}\right)$ indica diferencias significativas $(P \leq 0,05)$

por la RUV-B en todas las variedades ensayadas, la exposición a dicha radiación indujo un aumento significativo de su valor en comparación con las plantas que no fueron expuestas a la RUV-B (control). El incremento en NH también ha sido observado en variedades de quinoa cultivadas con y sin exclusión de la RUV-B solar en ensayos a campo bajo condiciones semicontroladas (Palenque et al., 1997; González et al., 2009).

Aunque la mayor parte de los estudios llevados a cabo bajo condiciones de exclusión de RUV-B solar y en presencia de RUV-B suplementaria artificial han puesto de manifiesto que las plantas mono y dicotiledóneas terrestres y acuáticas, responden negativamente en relación al número y tamaño de las hojas cuando son expuestas a dicha radiación (Feldheim \& Conner, 1996; Krizek et al., 1998; Zuk-Golaszewska et al., 2003). Algunas especies resultan insensibles a las variaciones de la RUV-B, por lo que no manifiestan ningún cambio en el valor de $\mathrm{NH}$ cuando son expuestas a niveles incrementados de RUV-B (Rousseaux et al., 2001; Stokłosa et al., 2012); mientras que otras exhiben aumentos significativos en el valor de NH al ser expuestas a dosis elevadas de RUV-B (Grammatikopoulos et al., 1998; Kumari et al., 2009), de un modo similar a lo observado en el presente trabajo. Al analizar el largo por ancho (LA) que es una variable estrechamente relacionada al área de la hoja, no se observaron cambios de significación inducidos por la RUV-B en ninguna de las variedades ensayadas, a excepción de la variedad CICA (Fig. 3). Este hecho podría indicar que la mayor inversión en carbono y energía que ocurre en las variedades de quinoa expuestas a la RUV-B para producir un mayor número de hojas, tendría por finalidad aumentar el número de sitios aptos para llevar a cabo la fotosíntesis y de este modo asegurar un crecimiento y productividad relativamente estables en condiciones de extrema irradiancia solar, tal como ocurre en los ambientes de alta montaña. En este sentido, el aumento del número de hojas pero no el de la superficie foliar individual, podría ser el resultado de una adaptación evolutiva de la quinoa a fin de sostener su capacidad de fotosíntesis teniendo en cuenta que su origen es la región altoandina sometida a una extrema irradiancia solar (superior a $3000 \mu \mathrm{mol} \mathrm{m} \mathrm{m}^{-2} \mathrm{~s}^{-1} \mathrm{en}$ horas de medio día). En ese sentido, un aumento en el número de hojas por planta traería aparejado un mayor solapamiento e interacción entre ellas, lo que lo que a su vez, ocasionaría una mayor dispersión y/o atenuación de la RUV-B incidente. En estas condiciones el daño fotooxidativo del fotosistema II (FSII) inducido por la RUV-B (Zlatev et al., 2012) sería menos intenso. Así, en las plantas expuestas a elevadas irradiancias un mayor número de hojas de menor superficie resultaría en una menor afectación de la capacidad fotosintética por la RUV-B, que en aquellas con un menor número de hojas pero de superficie mayor. No obstante, se requieren estudios adicionales con un mayor número de variables y con variedades de distintos orígenes a fin de confirmar la hipótesis aquí planteada.

$\mathrm{Al}$ analizar el AFE que es la variable relacionada al área y espesor tisular de la hoja, se observa que la misma resulta afectada por la RUV-B, ya sea, en forma positiva (aumento de AFE) como negativa (disminución de AFE) (Tabla 1); indicando para el primer caso que las variedades afectadas (Faro Roja, Kancolla) presentan una mayor área o un menor espesor de hoja que las plantas control, mientras 
que para las variedades afectadas negativamente (CICA, Ratuqui, Robura) que el área de la hoja es menor o el espesor foliar es mayor que el correspondiente a las plantas cultivadas en ausencia de RUV-B. Considerando que el parámetro largo por ancho (LA) prácticamente no mostró variaciones de significación podría asumirse que la variable afectada por la RUV-B corresponde al espesor tisular. Este hecho reviste cierta significación por cuanto el incremento del espesor foliar inducido por la RUV-B ha sido interpretado como una estrategia defensiva, ya que un mayor espesor contribuiría a reducir la penetración de la RUV-B en la hoja y así atenuar el potencial daño al aparato fotosintético (Kakani et al., 2003). Por el contrario en las variedades donde el AFE se incrementa por efecto de la RUV-B, podría asumirse que la disminución del espesor foliar se produce como consecuencia de la redistribución del esqueleto carbonado hacia la síntesis de compuestos protectores de UV-B tal el caso de los flavonoides y betalaínas (Ibdah et al., 2002). Cuando se considera la MFE como la variable afectada por la RUV-B, y considerando que dicho parámetro representa el "costo en carbono" que la planta invierte para producir $1 \mathrm{~cm}^{2}$ de hoja, surge que las variedades Faro Roja y Kancolla (mayor AFE) serían las menos afectadas por la RUV-B en términos metabólicos y de inversión de carbono (Milla \& Reich, 2007). El restante parámetro de crecimiento que fue medido en las cinco variedades de quinoa corresponde al diámetro de tallo (DT), y al igual que los anteriores también resultó diferencialmente afectado por la RUV-B. Globalmente los resultados de este trabajo junto a otros obtenidos a campo bajo condiciones naturales (González et al., 2009), ponen claramente de manifiesto que, al menos, una parte de los cultivares comerciales de quinoa actualmente disponibles, podrían resultar más o menos afectados por la RUV-B según cuál sea el valor de dicha radiación en el sitio de cultivo.

En consonancia con nuestros resultados, los datos disponibles en la literatura muestran que las respuestas de las plantas a la RUV-B son bastantes variables y aún contradictorios entre especies emparentadas e incluso entre cultivares dentro de una misma especie (Liu et al., 2004; Kalbina \& Strid, 2006; Robson \& Aphalo, 2012; Zlatev et al., 2012). Aunque no se ha encontrado una explicación totalmente convincente sobre la variabilidad observada en los efectos de la RUV-B, se ha sugerido que dicha variabilidad podría estar relacionada a la edad de la planta (Yao \& Liu, 2007), la procedencia de la especie estudiada (Lindroth et al., 2000) e incluso a la procedencia de su línea parenteral (Robson et al. 2014), o bien a su reloj biológico (ritmos circadianos) (Takeuchi et al., 2014). En ese sentido, se ha postulado que la adquisición y/o pérdida de determinados genes a lo largo de la evolución así como la regulación de su expresión, serían los principales responsables de los diferentes efectos inducidos por la RUV-B sobre el desarrollo de las plantas (Ulm \& Nagy, 2005; Liu et al., 2014). Durante mucho tiempo se aceptó que la RUV-B actuaba como un factor de estrés induciendo una plétora de respuestas morfológicas, fisiológicas y bioquímicas en las plantas (Caldwell et al., 1995) Sin embargo con el advenimiento de nuevas técnicas que permitieron eliminar $\mathrm{y} / \mathrm{o}$ reducir el nivel de RUV-B de la radiación solar que incide sobre las plantas (Rousseaux et al., 2004), condujeron a la obtención de nuevos datos sobre el comportamiento y respuestas de las plantas a la RUV-B (Prado et al., 2011). En ese contexto, se encontró que aunque muchas de las respuestas de las plantas a la RUV-B eran similares a las inducidas por otros factores de estrés sean éstos de origen biótico o abiótico, la RUV-B también inducía algunas respuestas que podrían considerarse como propias, exhibiendo una grado de variabilidad superior al de los otros estreses (Reboredo \& Lidon, 2012). Con posterioridad, el aislamiento y caracterización, entre otros, del fotorreceptor UVR8 involucrado en las respuestas fotomorfogénicas de las plantas (Favory et al., 2009; Jenkins, 2009), llevaron a postular si realmente la RUV-B debía considerarse un factor de estrés o un factor morfogénico; asumiendo que la misma tendría una influencia decisiva en el establecimiento y distribución de las especies en los ecosistemas naturales (Heijde \& Ulm, 2012; Robson et al., 2014). No obstante muchos cuestionamientos quedan aún por resolver para establecer fehacientemente el papel que desempeña la RUV-B en el desarrollo de la plantas. Entre tales cuestionamientos sobresale dilucidar si existe un umbral a partir del cual la RUV-B solar deja de ser un factor morfogénico natural para convertirse en un factor de estrés; y en caso de existir, cuál sería su dependencia de los factores endógenos (genéticos) propios de la especie así 
como de aquellos dependientes del propio individuo y del entorno donde crece.

\section{Conclusiones}

No obstante la necesidad de seguir avanzando en el estudio del efecto de la RUV-B, los aportes de este trabajo no sólo resultan de utilidad para avanzar en el conocimiento del comportamiento morfológico de las diferentes variedades de quinoa, frente a la RUV-B, sino también contribuir al afianzamiento de los programas dirigidos a expandir y/o reintroducir el cultivo de esta especie como fuente alternativa de nutrientes en áreas de baja potencialidad agrícola tal el caso de la alta montaña y zonas áridas y semiáridas con bajo nivel de precipitaciones y elevada radiación solar.

\section{Bibliografía}

BURNOUF-RADOSEVICH, M. 1988. Quinoa (Chenopodium quinoa Willd): A potential new crop. In: Bajaj, Y.P.S. (ed.), Biotechnology in Agriculture and Forestry, vol. 6, Crops II, pp. 386-404. Springer Verlag, Berlin.

CALDWELl, M., A.H. TERAMURA., M. TEVINI., J.F. BORNMAN., L.O. BJÖRN \& G. KULANDAIVELU. 1995. Effects of increased solar ultraviolet-radiation on terrestrial plants. Ambio 24: 166-173.

FAVORY, J.J., A. STEC., H. GRUBER., L. RIZZINI., A. ORAVECZ., M. FUNK., A. ALBERT., C. CLOIX., G.I. JENKINS., E.J. OAKELEY., H.K. SEIDLITZ., F. NAGY \& R. ULM. 2009. Interaction of COP1 and UVR8 regulates UV-B-induced photomorphogenesis and stress acclimation in Arabidopsis. EMBO J. 28: 591-601.

FELDHEIM, K. \& J.K. CONNER. 1996. The effects of increased UV-B radiation on growth, pollination success, and lifetime female fitness in two Brassica species. Oecologia 106: 284-297.

GONZÁLEZ, J.A., Y. KONISHI., M. BRUNO., M. VALOY \& F.E. PRADO. 2012. Interrelationships among seed yield, total protein and amino acid composition of ten quinoa (Chenopodium quinoa) cultivars from two different agroecological regions. J. Sci. Food Agric. 92: 1222-1229.

GONZÁLEZ, JA., Y. KONISHI., M. BRUNO., M. VALOY., ROSA., M. HILAL \& F.E. PRADO. 2010. Essential amino acids from different seed varieties of "quinoa" growing in Amaicha del Valle (Tucumán, Argentina). Biocell 34: 148.
GONZÁLEZ, J.A. \& F. PRADO. 1992. Germination in relation to temperature and different salt concentration in Chenopodium quinoa. Agrochimica 36: 101-107.

GONZÁLEZ, J.A., A. ROLDÁN., M. GALLARDO., T. ESCUDERO \& F.E. PRADO. 1989. Quantitative determinations of chemical compounds with nutritional value from Inca Crops: Chenopodium quinoa ("quinoa"). Plant Foods Hum. Nutr. 39: 331-337.

GONZÁLEZ, JA., M. ROSA., M.F. PARRADO., M. HILAL \& F.E. PRADO. 2009. Morphological and physiological responses of two varieties of a highland species UV-B in a lowland location. $J$. Photochem. Photobiol. Biology 96: 144-151.

GRAMMATIKOPOULOS, G., R. KAROUSOU., S. KOKKINI \& Y. MANETAS. 1998. Differential effects of enhanced UV-B radiation on reproductive effort in two chemotypes of Mentha spicata under field conditions. Austr. J. Plant Physiol. 25: 345-351.

HEIJDE, M. \& R. ULM. 2012. UV-B photoreceptormediated signalling in plants. Trends Plant Sci. 17: 230-237.

HILAL, M., M.F. PARRADO., M. ROSA., M.GALLARDO., E.M. MASSA., J.A. GONZÁLEZ \& F.E. PRADO. 2004. Epidermal lignin deposition in quinoa cotyledons in response to UV-B radiation. Photochem. Photobiol. 79: 205-210.

IBDAH, M., A. KRINS., H.K. SEIDLITZ., W. HELLER., D. STRACK \& T. VOGT. 2002. Spectral dependence of flavonol and betacyanin accumulation in Mesembryanthemum crystallinum under enhanced ultraviolet radiation. Plant Cell Environ. 25: 11451154.

JENKINS, G.I. 2009. Signal transduction in responses to UV-B radiation. Ann. Rev. Plant Biol. 60: 407-431.

KAKANI, V.G., K.R. REDDY., D. ZHAO \& K. SAILAJA. 2003. Field crop response to ultraviolet-B radiation: a review. Agric. For. Meteorol. 120: 191-218.

KALBINA, I. \& Å. STRID. 2006. Supplementary ultraviolet-B irradiation reveals differences in stress responses between Arabidopsis thaliana ecotypes. Plant Cell Environ. 29: 754-763.

KRIZEK, D.T., S.J. BRITZ \& R.M. MIRECKI. 1998. Inhibitory effects of ambient levels of solar UV-A and UV-B radiation on growth of cv. New Red Fire lettuce. Physiol. Plant.103: 1-7.

KUMARI, R., S. SINGH \& S.B. AGRAWAL. 2009. Effects of supplemental ultraviolet-b radiation on growth and physiology of Acorus calamus L. (sweet flag). Acta Biol. Cracoviensia, Ser. Bot. 51: 19-27.

LINDROTH, R.L., R.W. HOFMANN., B.D. CAMPBELL., W.C. MCNABB \& D.Y. HUNT. 2000. Population differences in Trifolium repens L. response to ultraviolet-B radiation: foliar chemistry and consequences for two lepidopteran herbivores. 
Oecologia 122: 20-28.

LIU, L., S. GREGAN., C. WINEFIELD \& B. JORDAN. 2014. From UVR8 to flavonol synthase: UV-Binduced gene expression in Sauvignon blanc grape berry. Plant Cell Environ. Doi: 10.1111/pce.12349.

LIU, Q., T.V. CALLAGHAN \& Y. ZUO. 2004. Effects of elevated solar UV-B radiation from ozone depletion on terrestrial ecosystems. J. Mt. Sci. 1: 276-288.

MILLA, R. \& P.B. REICH. 2007. The scaling of leaf area and mass: the cost of light interception increases with leaf size. Proc. Royal Soc.London B. 274: 2109-2114.

PALENQUE, E.R., M. ANDRADE., J.A. GONZÁLEZ., R. FORNO., V. LAIRANA., F.E. PRADO., J.C. SALCEDO \& S. URCULLO. 1997. Efectos de la radiación ultravioleta sobre la quinoa (Chenopodium quinoa Willd.). Revista Boliv. Fisiol. 3: 120-128.

PRADO, F.E., J.L. FERNÁNDEZ-TURIEL., M. BRUNO., M. VALOY., M. ROSA \& J.A. GONZÁLEZ. 2010. Mineral content in seed of quinoa varieties growing in Amaicha del Valle (Tucumán, Argentina). Biocell 34:157.

PRADO, F.E., M. ROSA., C. PRADO., G. PODAZZA., R. INTERDONATO., J.A. GONZÁLEZ \& M. HILAL. 2011. UV-B radiation, its effects and defense mechanisms in terrestrial plants. In: Parvaiz, A. \& M.N.V. Prasad (eds.), Environmental Adaptations and Stress Tolerance of Plants in the Era of Climate Change, pp. 57-83. Springer Science+Business Media, New York.

REBOREDO, F. \& J.C. LIDON. 2012. UV-B radiation effects on terrestrial plants - A perspective. Emir. J. Food Agric. 24: 502-509.

RISI, C. \& N.W. GALWEY. 1984. The Chenopodium grains in the Andes: Inca crops for modern agriculture. Adv. Appl. Biol. 10: 145-216

ROBSON, T.M. \& P.J. APHALO. 2012. Species-specific effect of UV-B radiation on the temporal pattern of leaf growth. Physiol. Plant. 144: 146-160.

ROBSON, T.M., K. KLEM., O. URBAN \& M.A.K. JANSEN. 2014. Re-interpreting plant morphological responses to UV-B radiation. Plant Cell Environ. Doi: $10.1111 /$ pce. 12374 .

ROUSSEAUX, M.C., S.D. FLINT., P.S. SEARLES \& M.M. CALDWELL. 2004. Plant responses to current solar ultraviolet-B radiation and to supplemented solar ultraviolet-B radiation simulating ozone depletion: an experimental comparison. Photochem. Photobiol. 80: 224-230.

ROUSSEAUX, M.C., A.L. SCOPEL., P.S. SEARLES., M.M. CALDWELL, O.E. SALA \& C.L. BALLARE. 2001. Responses to solar ultraviolet-B radiation in a shrub-dominated natural ecosystem of Tierra del Fuego (southern Argentina). Global Change Biol.7: 467-478.

RUFFINO, A.M.C., M. ROSA., M. HILAL., J.A. GONZÁLEZ \& F.E. PRADO. 2010. The role of cotyledon metabolism in the establishment of quinoa (Chenopodium quinoa) seedlings growing under salinity. Plant Soil 326: 213-224.

SIRCELJ, M.R., M.D. ROSA., M.F. PARRADO., J.A. GONZÁlEZ., M. HILAL \& F.E. PRADO. 2002. Ultraestructural and metabolic changes induced by UV-B radiation in cotyledons of quinoa (Chenopodium quinoa Willd). Biocell 26: 180.

STOKŁOSA, A., H. MADANI \& M.K. UPADHYAYA. 2012. Response of hoary alyssum (Berteroa incana L.) to UV-B radiation. Acta Agrobot. 65: 67-72.

TAKEUCHI, T., L. NEWTON., A. BURKHARDT., S. MASON \& E.M. FARRE. 2014. Light and the circadian clock mediate time-specific changes in sensitivity to UV-B stress under light/dark cycles. $J$. Exp. Bot. 65: 6003-6012.

ULM, R. \& F. NAGY. 2005. Signalling and gene regulation in response to ultraviolet light. Curr. Opin. Plant Biol. 8: 477-482.

YAO, X. \& Q. LIU. 2007. Responses in growth, physiology and nitrogen nutrition of dragon spruce (Picea asperata) seedlings of different ages to enhanced ultraviolet-B. Acta Physiol. Plant. 29: 217-224.

ZLATEV, Z.S., F.J.C. LIDON \& M. KAIMAKANOVA. 2012. Plant physiological responses to UV-B radiation. Emir. J. Food Agric. 24: 481-501.

ZUK-GOLASZEWSKA, K., M.K. UPADHYAYA \& J. GOLASZEWSKI. 2003. The effect of UV-B radiation on plant growth and development. Plant Soil Environ. 49: 135-140.

Recibido el 4 de enero de 2015, aceptado el 29 de abril de 2015 . 
